\title{
Development of laser-excited, fluorometric assays for the monitoring of toxic chemical exposure
}

\author{
C.A. Burtis, B. Z. Egan, N. E. Lee \\ Chemical Technology Division, Oak Ridge National Laboratory, Oak Ridge, \\ Tennessee 37831 , USA
}

\begin{abstract}
The microsomal mixed-function oxidase (MFO) enzyme system is involved in the metabolism of various chemical compounds. Polycylic aromatic hydrocarbons and related compounds are metabolized by the cytochrome $\mathrm{P}-448$ enzyme system, which contains MFOs. Induction of this MFO activity by toxic chemicals may be useful as an indicator of the toxicity of the inducer material. A centrifugal analyzer equipped with an argon-ion laser light source was used to quantitate cytochrome $\mathrm{P}-448$ activity in mouse liver microsomes. The activity was induced by exposing the animals to different doses of liquids derived from a coalliquefaction process. The P-448 MFO activity was determined kinetically by measuring the rate at which the highly fluorescent compound, resorufin, produced by oxidation of 7-ethoxy resorufin substrate, was formed. The 514.5-nm 1aser excitation beam was directed with a fiber optic bundle from the laser to the cuvettes of a specially designed rotor. The emitted fluorescence, filtered through a 560-nm cut-on filter, was monitored at $90^{\circ}$ to the incident beam. A rotor, consisting of a black, acrylic plastic center body with transparent acrylic cuvette windows, was designed and fabricated to improve the fluorescence measurements. The use of a laser excitation source allows very low MFO activities to be measured; femtomole quantities of resorufin can be determined.
\end{abstract}

\section{INTKODUCTION}

Xenobiotic compounds are metabolized and detoxified by the microsomal enzyme system known collectively as cytochrome mixed-function oxidases (MFOs). This oxidative metabolic process can also result in the formation of active intermediates that can react with cellular macromolecules such as DNA to form potentially toxic and carcinogenic adducts (1). Consequently, the measurement of cytochrome MFO activity has been used to assess the biological activity and potential toxicity of compounds or mixtures of compounds (2). Ioannides and coworkers (3) have listed several compounds that can induce the activity of P-448 MFO, a subset of the MFO system which is preferentially induced by polycyclic a romatic hydrocarbons (PAHs) and other related compounds.

Various methods have been developed for measuring MFO activity, including the measurement of fluorescence (4) or radioactivity (5) of extracted metabolites. However, these methods are time-consuming, laborious and, in general, lack the attributes of an automated technique. Alternately simple enzyme methods have been developed that utilize a variety of specific substrates to measure individual enzymes in the MFo system (6). For example, Burke and Mayer (7) have synthesized 7-ethoxyresorufin (7-ethoxyphenoxazone) and utilize it as a substrate in a fluorometric enzyme assay that can be used to measure $\mathrm{P}-448 \mathrm{MFO}$ activity.

We have adapted this fluorometric technique for use with a centrifugal analyzer (8) equipped with an argon-ion laser (9). This approach has allowed us to automate and measure very low levels of $\mathrm{P}-448$ activity. In this report we will describe this system, present the type of data obtained with it, and discuss its automation capabilities.

\section{MEASUREMENT OF P-448 MFO ACTIVITY}

Inst rumentation

Figure 1 shows a schematic diagram of the analytical system used in this study. Fluorescence was measured in a centrifugal analyzer equipped with a 5-W argon-ion laser (Spectra Physics, Mountain View, CA 94042) as the excitation source. The 514.5-nm output of the laser was directed to the cuvettes of the spinning rotor through a fiber-optic bundle at a $90^{\circ}$ angle to the detector. The emitted fluorescence was filtered through a 


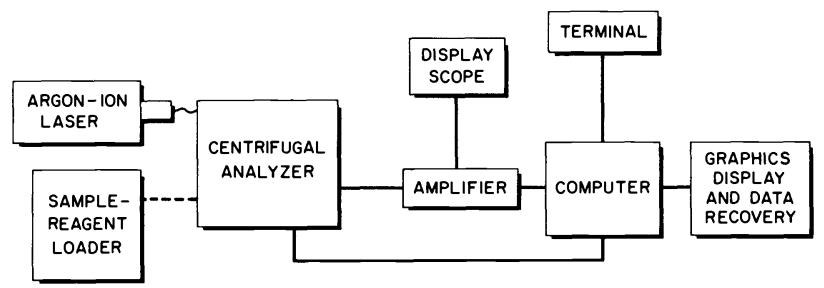

Fig. 1. Schematic of the analytical system used for fluorescence measurements. Dotted line indicates that rotors are loaded off-line and placed into analyzer for processing.

560-nm cut-on (maximum transmission above $560 \mathrm{~nm}$ ) filter (Ditric Optics, Inc., Marlboro, MA 01752) located between the rotor and the photomultiplier tube. The fluorescence excitation and emission spectra are shown in Fig. 2.

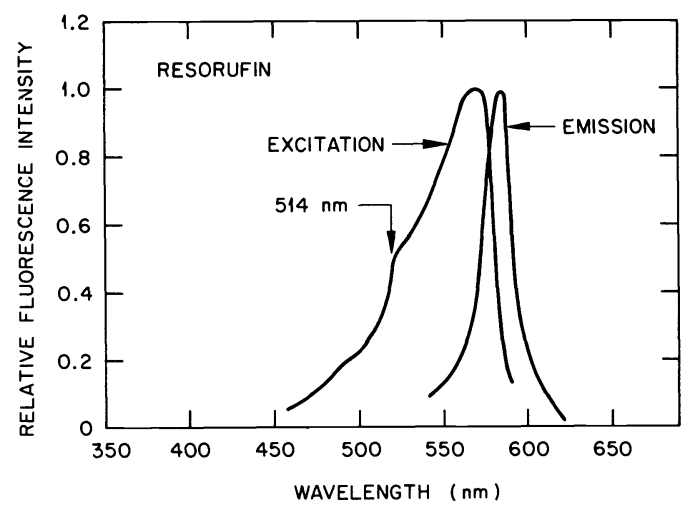

Fig. 2. Fluorescence excitation and emission spectra for resorufin. Arrow indicates where argon-ion laser produces excitation energy $(514.5-\mathrm{nm})$.

For data acquisition and processing, a PDP-11V03 computer (Digital Equipment Corp., Marlboro, MA 01752) was used that was equipped with dual diskette drives and a CKT (Model 4006-1, Tektronix, Beaverton, OR 97005). The system was run with the kT-11 operating system and included the high-level FOCAL language enhanced with assembly language routines.

A rotor, consisting of a black, acrylic plastic center body with transparent acrylic cuvette windows, was designed and fabricated to reduce interference from fluorescence and scattered light in adjacent cuvettes. Buffer solution in cuvette 1 was used to correct for dark current and background fluorescence. A standard resorufin reference solution was used in cuvette 2 for dynamic referencing (10). Aliquots of samples and reagent were automatically loaded into their respective chambers of the rotor using a mechanical pipetting device (11).

Animal treatment

All enzyme samples we re prepared for mouse livers. Test substances were suspended in corn oil and administered by intra-peritoneal injection. When 3-methylcholanthrene was used to induce enzyme activity, it was injected in volumes equivalent to $10 \mathrm{~mL} / \mathrm{kg}$ of body weight at a dose of $25 \mathrm{mg} / \mathrm{kg}$ of body weight. The animals we re sacrificed $48 \mathrm{~h}$ later.

\section{Microsome preparation}

The liver of each animal was promptly removed and washed in $\operatorname{cold}\left(4^{\circ} \mathrm{C}\right)$ potassium phosphate buffer ( $10 \mathrm{mmol} / \mathrm{L}, \mathrm{pH} 7.4)$ containing $150 \mathrm{mmol} / \mathrm{L}$ of $\mathrm{KCl}$. It was then minced and homogenized with $2 \mathrm{~mL}$ of buffer per gram (wet weight) of tissue. The homogenates we re centrifuged $(9000 \times \mathrm{g}, 20 \mathrm{~min}$; Sorvall RC-5B, Du Pont, Newton, CT 06470). A 170- $\mu \mathrm{L}$ aliquot of the resulting "S-9" supernate was centrifuged at $105000 \times \underline{g}$ (Airfuge; Beckman Instruments, Fullerton, CA 92634) for $10 \mathrm{~min}$ to recover the microsome pellets. These pellets were subsequently resuspended in $50 \mu \mathrm{L}$ of tris(hydroxymethy1)-aminomethane buffer (100 mmol/L, $\mathrm{pH} \mathrm{7.4,} \mathrm{and} \mathrm{containing} 1 \mathrm{mmol}$ of EDTA and $100 \mathrm{~mL}$ of glycerol per liter) and stored at $-70^{\circ} \mathrm{C}$.

\section{Enzyme assay}

Based on a previous optimization study (9), the final reaction mixture contained, per

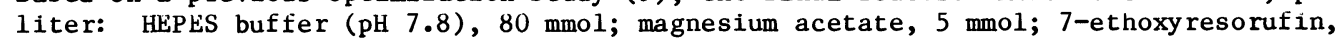
$1.0 \mu \mathrm{mol} ; \mathrm{NADPH}, 250 \mu \mathrm{mol}$; and EDTA, $100 \mu \mathrm{mol}$. The concentration of total protein ranged $f$ rom 0.2 to $100 \mathrm{mg} / \mathrm{L}$, depending on the enzyme activity of the sample. Sources for the individual reagents have been listed previously (9). 
After the reaction $\left(\right.$ at $30^{\circ} \mathrm{C}$ ) was started, fluorescence measurements we re made at fixed intervals during several minutes, and the rate of resorufin formation was calculated from the initial slope of the curve.

\section{Representative data}

The use of the argon-ion laser as a light source increases the excitation intensity and, consequently, the intensity of the fluorescent emission. Thus, the signal-to-noise level of the system increases, which improves precision of the fluorescent measurements and increases sensitivity. For example, Fig. 3 demonstrates the improvement in precision and linearity obtained by using a laser light source (a) to measure the fluorescence of standard resorufin solutions. In this figure, the oscilloscope displays the fluorescence signals resulting from resorufin solutions ranging in concentration from 1 to $5 \times 10^{-9} \mathrm{M} / \mathrm{L}$. At these levels, intercuvette precision was approximately $\pm 1.5 \%$. For comparison, the fluorescent signals are also shown when a tungsten-halogen monochromator (b) was used as the light source. It should be noted that since the total volume for each cuvette is $125 \mu \mathrm{L}$, the data shown in Fig. 3 correspond to approximately 100-700 fmol of resorutin per cuvette. For analytical purposes, this degree of sensitivity translates to a capability for measuring enzyme activities of less than $1 \mathrm{U} / \mathrm{g}$ in an aliquot containing less than $10 \mathrm{ng}$ of protein.

In Fig. 4, both light sources were used to measure the MFO activity in aliquots representing $100 \mathrm{ng}$ of protein of a liver microsome sample from animals injected with 3 -methylcholanthrene. As can be seen, both produced linear relationships between fluorescence produced with time; however, precision was better with the argon-ion laser (a) than with the tungsten-halogen monochromator (b).

In a five-day study, replicate aliquots of a reference sample we re analyzed and estimates of the within-day and day-to-day precision were determined to be $\pm 2.3 \%$ and $\pm 7.0 \%$, respectively.

\section{Analytical applications}

We have utilized the fluorometric 7-ethoxyresorufin assay to measure MFO activity in a variety of treated and control animals $(2,9)$. For example, a dose/response relationship was demonst rated for animals that had been treated with 3 -methylcholanthrene at levels ranging from $5-25 \mathrm{mg} / \mathrm{kg}$ of body weight. Parallel experiments also demonstrated that $\mathrm{P}-448$ activity was preferentially induced by 3 -methylcholanthrene relative to phenobarbitol, an inducer of the P-450 MFO system.
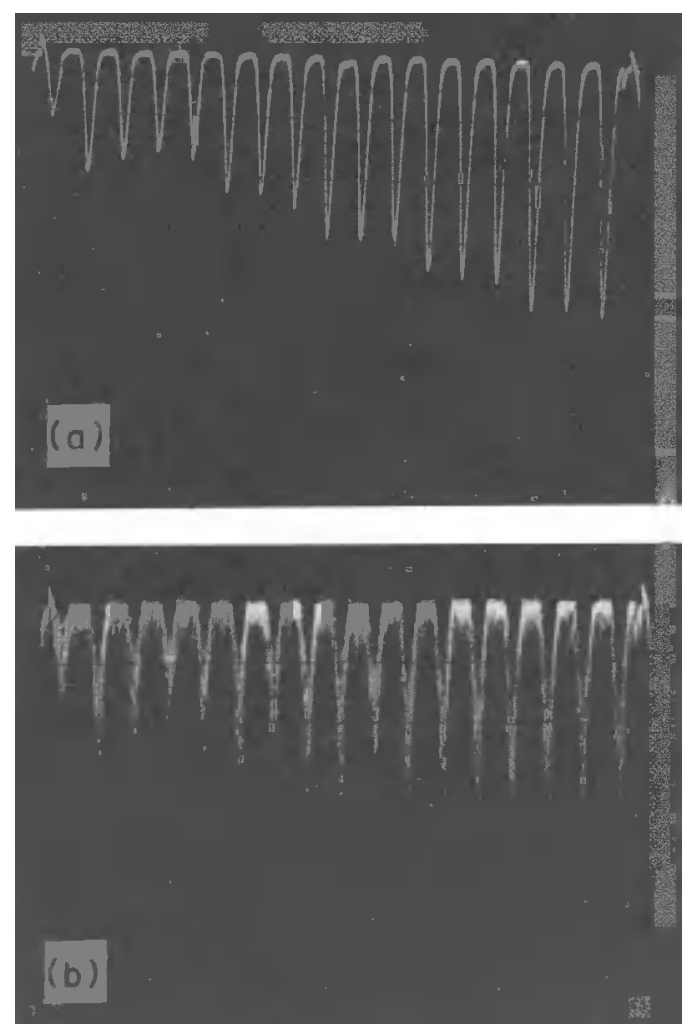

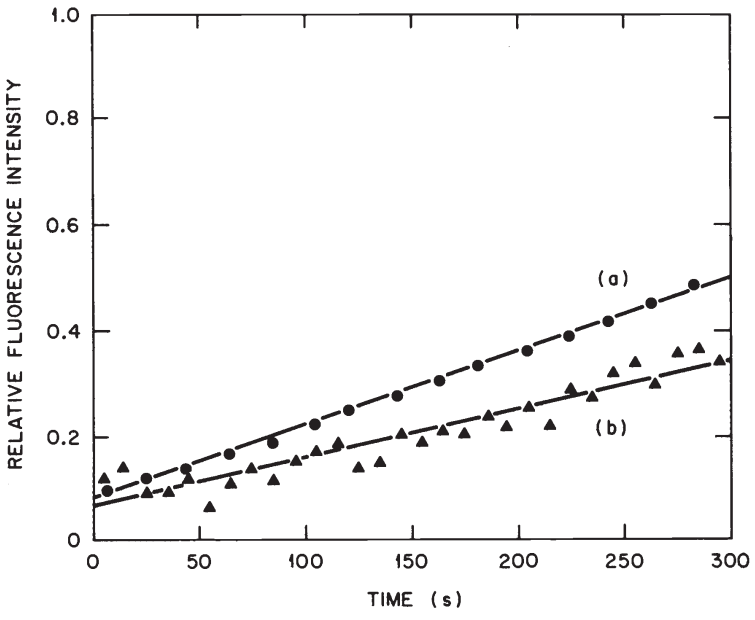

Fig. 4. P-448 MFO activity measurements obtained with either an argon-ion laser (a) or tungsten/halogen monochromator (b) as the light source. Total protein for each was approximately $100 \mathrm{ng}$.

Fig. 3. Oscilloscope displays of fluorescence signals generated by standard solutions of - resorufin ranging in concentration from 1-5 mol/L. Top and bottom displays were obtained using an argon-ion laser and tungsten/halogen monochromator as excitation sources. 
In a related series of experiments, animals were treated with a natural petroleum blend, a coal liquid produced by the Char-Oil-Energy Development (COED) process and a coal liquid produced by the Synthoil process. All three liquids induced P-448 activity, with Synthoil liquid being the most potent inducer, followed by the COED liquid and the natural petroleum blend. The induction of P-448 MFO activity also parallels the concentration of benzo $(\alpha)$ py rene present in each of these liquids (12). In separate studies, the carcinogenicity (13) and mutagenicity (14) were determined and their potencies found to rank in the same order as the MFO inductions (i.e., Synthoil > COED > blend).

To evaluate other compounds for their ability to induce P-448 MF0 activity, a series of transformer liquids were tested that contained polychlorinated biphenyls (PCBs), a class of compounds of known toxicity (15). As expected, a dose/response was observed between P-448 MFO activity and various $\mathrm{PCB}$ mixtures (2).

To utilize the increased sensitivity afforded by the use of the argon-ion laser, a series of experiments were conducted in which animals we re topically treated and liver P-448 MFO activity determined (9). Even under these experimental conditions, which required dermal adsorption followed by systemic dilution and transport to the liver, differential P-448 MFO inductions were observed for various coal liquids.

In ongoing experiments, we are treating animals and attempting to measure $\mathrm{P}-448 \mathrm{MFO}$ activity in microsomal fractions from various tissues such as the lung, spleen, thymus, and also from lymphocytes.

SUMMARY

A fluorometric method has been developed for measuring P-448 MFU activity in animals treated with toxic chemicals. The method has been adopted and optimized for use with a centrifugal analyzer equipped with an argon-ion laser. The resultant system has the attributes of an automated technique. For example, its evaluation and application has demonstrated that it provides acceptable analytical performance, as it is an extremely sensitive system and can provide meaningful analytical measurements at the fmol level. The system is time effective, as all of the time-requiring steps (i.e., sample loading, sample analysis, and data acquisition and processing) are all performed by machines with little operator involvement required. With this system, approximately 60 samples can be processed and analyzed daily. Using techniques that require a separation step, 2 to 3 days would be required to process and analyze the same number of samples. Centrifugal analyzers require small volumes of sample and reagent for operation. For example, the analyzer used in this study requires only 5 to $10 \mu \mathrm{L}$ of sample, which is analyzed in a total reaction volume of $125 \mu \mathrm{L}$ per cuvette. Thus, sample economy and reduced reagent costs are features of these instruments that result in their having a reputation of being very cost-effective machines to operate.

Acknowledgement - This work was supported by the Office of Health and Environmental Research, U.S. Department of Energy, and was performed at the Oak Ridge National Laboratory, which is operated by Martin Marietta Energy Systems, Inc., under Contract No. DE-ACO5-840R21400.

REFERENCES

1. H. V. Gelboin, N. Kinoshita, and F. J. Wiebel, Fed. Proc., Fed. Am. Soc. Exp. Biol. 31, 1298-1309 (1972).

2. W. D. Bostick, J. Kao, J. M. Holland, and J. E. Mrochek, Clin. Chem. 27, 1516-1523 (1981).

3. C. Ioannides, P. Y. Lum, and P. V. Parke, Xenobiotica 14, 119-137 (1984).

4. D. W. Nebert and H. V. Gelboin, J. Biol. Chem. 243, 6242-6249 (1968).

5. D. Kupfer and J. Rosenfeld, Drug. Metab. Dispos. 1, 760-765 (1973).

6. M. J. Coon and A. V. Persson, Enzymatic Basis of Detoxification, p. 121, Academic Press, New York (1980).

7. M. D. Burke and R. T. Mayer, Drug Metab. Dispos. 2, 583-588 (1974).

8. J. E. Mrochek, C. A. Burtis, W. F. Johnson, M. L. Bauer, D. G. Lakomy, K. K. Genung, and C. D. Scott, Clin. Chem. 23, 1416-1426 (1977).

9. B. Z. Egan, N. E. Lee, C. A. Burtis, J. Y. Kao, and J. M. Holland, Clin. Chem. 29 , 1616-1619 (1983).

10. T. O. Tiffany, M. B. Watsky, C. A. Burtis, and L. H. Thacker, Clin. Chem. 19, $871-882$ (1973).

11. C. A. Burtis, W. F. Johnson, and J. B. Overton, Anal. Chem. 46, 786-789 (1974).

12. J. L. Epler, et al., Mutat. Res. 57, 265-276 (1978).

13. J. M. Holland, et al., J. Occup. Med. 21, 614-618 (1979).

14. M. R. Guerin, et al., Environ. Res. 23, 42-53 (1980).

15. R. D. Kimbrough, CRC Crit. Rev. Toxicol. 2, 445-498 (1974). 